\title{
Einführung: Qualität und Wirtschaftlichkeit - ein Widerspruch?
}

\section{K. Dorfmeister OVE}

Online publiziert am 7. November 2014

(C) Springer Verlag Wien 2014

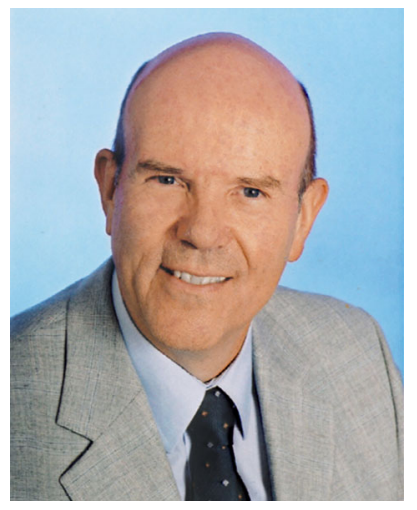

Dipl.-Ing. Dr. Karl Dorfmeister
Es ist fast schon gleichgültig, in welchen Lebenslagen man sich befindet, immer und überall ist eine der ersten Fragen: "Lohnt es sich?" oder "Geht es noch günstiger?". Der Druck des Rechenstifts scheint stets gegenwärtig und immer bedeutender zu sein. Nicht gemeint ist in diesem Zusammenhang die Entwicklung in so manchen Bereichen des Alltags, wo vieles durch Verbesserung der Fertigungsmethoden und hohe Produktionsziffern billi-

ger und somit für viele erschwinglich geworden ist.

Vermutlich hatte schon jeder einmal das Gefühl, dass vor Jahren zwar so manches teurer, aber die Lebensdauer der Produkte doch eindeutig länger und auch die Qualität eine höhere war. Oder soll man gar durch zunehmende Störungsanfälligkeit verleitet werden, eine Erneuerung vorzunehmen? In letzter Zeit wurden derartige Gedankenansätze immer wieder von den Medien aufgegriffen, der richtige Beweis dafür oder dagegen konnte letztendlich nicht erbracht werden. Dass Qualität und Preis in irgendeiner Form ja doch zusammenhängen müssen, tritt spätestens dann ins Bewusstsein aller, wenn irgendwelche spektakulären Rückrufaktionen, egal in welcher Branche, so manchen renommierten Hersteller in Bedrängnis bringen.
Ein Sprichwort sagt: "Wer billig kauft, kauft teuer". Oft heißt es auch: „Solide Qualität hat eben ihren Preis!“ Im schnelllebigen Konsumzeitalter sind diese Denkansätze für das Erreichen hoher Produktionsziffern vielleicht nicht mehr so gegenwärtig - gilt das am Ende nun auch schon für langlebige Investitionsgüter? Bewegen wir uns in der Energiewirtschaft womöglich gar bereits auf dem Weg zur Wegwerfgesellschaft?

Da eine sichere Stromversorgung die Grundlage für alle Bedürfnisse der heutigen Gesellschaft darstellt, ist die Beantwortung dieser Fragen von besonderer Bedeutung, und die Österreichische Gesellschaft für Energietechnik (OGE) im Österreichischen Verband für Elektrotechnik will mit ihrer 52. Fachtagung diese Antworten geben. Hochrangige Vertreter der Wissenschaft und Forschung, der einschlägigen Industrie und der Energiewirtschaft zeigen mit ihren äußerst interessanten Beiträgen auf, wie die (gewohnte) Qualität unter dem wirtschaftlichen Druck aufrechterhalten werden kann; denn wenn es nicht gelingt, die bisherigen Anforderungen an die Qualität zu erfüllen, sind letztendlich unangenehme Folgen für die gesamte Gesellschaft nicht auszuschließen.

Für die Unterstützung dieser äußerst interessanten Fachtagung ergeht der Dank der OGE an dieser Stelle an die Wels Strom $\mathrm{GmbH}$ als Hauptsponsor sowie an folgende Unternehmen und öffentliche Stellen: ABB AG, ALSTOM Austria GmbH, ANDRITZ HYDRO GmbH, Austrian Power Grid AG, Bundesministerium für Verkehr, Innovation und Technologie, Eaton Industries (Austria) $\mathrm{GmbH}$, Energie AG Oberösterreich, Energie-Control GmbH, Energienetze Steiermark $\mathrm{GmbH}$, Österreichische Forschungsförderungsgesellschaft GmbH, Pöyry Energy GmbH, Schneider Electric, SIEMENS AG Österreich, Sprecher Automation GmbH, TIWAG-Tiroler Wasserkraft AG, VERBUND Hydro Power GmbH und Wiener Netze GmbH.

Dorfmeister, Karl, Österreichische Gesellschaft für Energietechnik im OVE, Eschenbachgasse 9, 1010 Wien, Österreich (E-Mail: k.dorfmeister@ove.at) 